Proc. of the XI Int. Conf. - Ion Implantation and other Applications of Ions and Electrons, Kazimierz Dolny 2016

\title{
Radiation Degradation of Bipolar Transistor Current Gain
}

\author{
S.A. Miskiewicz ${ }^{a}$, A.F. Komarov ${ }^{a}$, F.F. Komarov ${ }^{a, *}$, G.M. Zayats $^{b}$ And S.A. Soroka $^{c}$ \\ ${ }^{a}$ Institute of Applied Physics Problems, 7 Kurchatov Str., 220045 Minsk, Belarus \\ ${ }^{b}$ Institute of Mathematics, National Academy of Sciences of Belarus, 11 Surganov Str., 220072 Minsk, Belarus \\ ${ }^{c}$ Belmicrosystems, Integral, 121 Kazintsa Str., 220108 Minsk, Belarus
}

\begin{abstract}
Spatial distribution of nonequilibrium minority charge carriers in bipolar transistors before and during the radiation exposure is described. Radiation-induced changes in the input and output characteristics and the current gain under the ${ }^{60} \mathrm{Co} 1.2 \mathrm{MeV} \gamma$-rays were calculated. It was shown that the collector current and current gain steadily fall due to irradiation in the considered range in the dose range $0-7 \times 10^{5} \mathrm{rad}$. The simulation results correlate well with the experimental data obtained at the Research and Production Corporation "Integral".
\end{abstract}

DOI: 10.12693/APhysPolA.132.288

PACS/topics: radiation, numerical simulation, bipolar transistors, lifetime, minority charge carriers, recombination, current gain

\section{Introduction}

Currently bipolar transistors are widely used in the micro- and nanoelectronics. As a part of equipment for military purposes as well as for the spacecrafts and nuclear facilities, transistors need to be highly radiation tolerant.

To improve the radiation resistance of $\mathrm{Si}$ devices, it is very important to understand the effects of radiation on these devices. Generally, the effects of radiation on semiconductor devices can be divided into three classes: displacement damage effects, total ionizing dose effects, and single event effects $[1,2]$. The first type is caused by defects in the semiconductor lattice that are accumulated due to the exposure to a certain fluence of high-energy particles. These defects form extra energy levels in the band gap and degrade the electrical characteristics of devices. The second type of effects occur when fixed charges and/or interface traps in the MOS devices are accumulated due to a certain dose of high-energy photons, such as gamma-rays. They also change the electrical characteristics of MOS devices. The third type effects are caused by single high-energy heavy particles passing through semiconductor devices. The single particle generates high density of electron-hole pairs, and undesirable charges flow through the devices. Such charges cause temporal errors in microprocessors or memory devices as well as catastrophic failures in power devices.

Bipolar transistors are mostly affected by the displacement damage generated by the first type effects. At large cumulative irradiation this mechanism was found to be responsible for the decrease of the gain of bipolar transistors mostly as a result of the decrease of the minority-carrier lifetime in the transistor base and for the degradation of the series-noise performance of chargesensitive-preamplifiers with bipolar junction transistors

\footnotetext{
*corresponding author; e-mail: KomarovF@bsu.by
}

in the input stage mainly due to the increase of the base spreading-resistance of the transistors [3, 4]. Calculating the possible changes in the device characteristics in the radiation environment allows to avoid the costly and long lasting experimental test series.

In this paper the physical and mathematical models of radiation degradation of integral $p-n-p$ bipolar transistor operating in the common-emitter amplifying mode under the ${ }^{60} \mathrm{Co} 1.2 \mathrm{MeV} \gamma$-ray irradiation are developed. The impact of neutron flow on the characteristics of bipolar transistors operating in the same mode was simulated in our earlier paper [5].

The models are based on a real topology of devices designed and manufactured by the "Integral" company. The software based on the developed models allows us to calculate the spatial distribution of minority-carriers, the recombination losses, as well as determine the changes in the input and output currents and the current gain. The radiation-induced changes in the output characteristics and the current gain have been simulated and the results are presented and discussed.

\section{The model}

The radiation degradation of bipolar transistors is caused mostly by the generation of radiation defects in the semiconductors i.e. the recombination centers reducing the lifetime of minority charge carriers in the device workspaces.

The model is based on the topology of the real integral transistor where the acceptor concentration in the device emitter is two-order higher than the donor concentration in the base so the emitter-base junction is asymmetrical. This indicates that the electron injection from the base to the emitter is insignificant as compared with the hole injection from the emitter to the base.

Thus the model of space-time evolution of minority charge carriers in the base of $p-n-p$ transistor includes the hole equation of continuity [6]: 


$$
\begin{gathered}
\frac{\partial p(x)}{\partial t}=-\frac{p(x)-p_{n}(x)}{\tau_{p}(x)}+D_{p} \frac{\partial^{2} p(x)}{\partial x^{2}} \\
-\mu_{p} E(x) \frac{\partial p(x)}{\partial x}-p(x) \mu_{p} \frac{\partial E(x)}{\partial x}
\end{gathered}
$$

with the border conditions

$$
p\left(W_{A}\right)=0, \quad p(0)=p_{n}(0) \exp \left(\frac{q U_{E B}}{k T}\right),
$$

the electric field intensity

$$
E(x)=-\frac{k T}{q} \frac{1}{N(x)} \frac{\mathrm{d} N(x)}{\mathrm{d} x},
$$

the modulation of collector junction width

$$
W_{C}=W_{C 0} \sqrt{1-\frac{U_{C}}{U_{d c}}},
$$

the dependence of hole lifetime on the radiation dose

$$
\frac{1}{\tau_{\Phi}}=\frac{1}{\tau_{0}}+k_{\tau} \Phi
$$

the density of emitter and collector currents

$$
J_{p \epsilon}=\frac{\mathrm{d} p(0)}{\mathrm{d} x} q D_{p}, \quad J_{p \kappa}=\frac{\mathrm{d} p\left(W_{A}\right)}{\mathrm{d} x} q D_{p},
$$

where $p$ is the nonequilibrium hole concentration, $p_{n}$ is the equilibrium hole concentration, $\tau_{p}$ is the hole lifetime, $D_{p}$ is the hole diffusion coefficient, $\mu_{p}$ is the hole mobility, $k$ is the Boltzmann constant, $q$ is the electron charge, $T$ is the absolute temperature, $N(x)$ is the donor concentration, $U_{E B}$ is the base voltage, $W_{A}$ is the active base thickness, $\tau_{0}$ is the initial hole lifetime, $\tau_{\varphi}$ is the radiation-induced hole lifetime, $\Phi$ is the radiation dose, $k_{\tau}$ is the factor of radiation changes of hole lifetime, $W_{C 0}$ is the initial width of collector junction, $U_{d c}$ is the base-collector potential barrier, $U_{C}$ is the collector voltage. The $X$-coordinate starts at the border between the emitter space-charge region and the base.

Considering the stationary process, Eq. (1) can be transformed to the following one describing the spatial distribution of hole charge:

$$
\begin{aligned}
& \frac{p(x)-p_{n}(x)}{\tau_{p}(x)}-D_{p} \frac{\partial^{2} p(x)}{\partial x^{2}}+\mu_{p} E(x) \frac{\partial p(x)}{\partial x} \\
& +p(x) \mu_{p} \frac{\partial E(x)}{\partial x}=0 .
\end{aligned}
$$

The equilibrium hole concentration in the $n$-base in accordance with [7] is

$$
p_{n}(x)=\frac{n_{i}^{2}}{n_{n}(x)},
$$

where $n_{i}$ is the intrinsic carrier concentration in Si, $n_{n}(x)$ is the electron concentration in the base equal to the dopant (phosphorus) concentration $N(x)$. Thus, one of the border conditions is transformed to

$$
p(0)=\frac{n_{i}^{2}}{N(0)} \exp \left(\frac{q U_{E B}}{k T}\right) .
$$

Solving (7), in accordance with Eqs. (2)-(5), (9), delivers the spatial distribution of the nonequilibrium holes $p(x)$ in the operating device regions for any doses of radiation exposure. In some cases [6] the calculation of the spatial distribution of $p(x)$ in the active base can be simplified

$$
p(x)=p(0)\left(1-\operatorname{ch} \frac{x}{L} / \operatorname{ch} \frac{W_{A}}{L}\right),
$$

where $L$ is the hole diffusion length

$$
L=\sqrt{D_{p} \tau_{p}} .
$$

In the passive base, the spatial distribution of $p(x)$ can be found by

$$
p(x, y)=n_{i}^{2}\left(\frac{1}{N_{p}}\right) \exp \left(\frac{q U_{E B}}{k T}\right) \exp \left(\frac{-y}{L}\right) .
$$

The solution of Eqs. (10) and (12) with Eqs. (11), (9), (5) and (4) is based on the iteration algorithm and provides the base distribution of the nonequilibrium minor charge carriers $p(x)$. The calculated spatial distribution of $p(x)$ allows to calculate the emitter and collector currents using Eq. (6).

Full emitter and collector currents are the integral of Eq. (6) over the areas of the appropriate junctions. The base current is the difference of the emitter and collector currents. The current gain of common-emitter mode is a ratio of the collector and base currents.

The factor $k_{\tau}$ depends, in general, on the transistor topology, the hole injection level, the energy and type of radiation particles, irradiation mode. Its exact value can be assessed during the experimental investigations only. The trial-and-error method assessing the numerical value of factor $k_{\tau}$ was applied to fit the developed model to the experimental results.

\section{Results and discussion}

The numerical simulations of spatial distribution of the nonequilibrium holes and the radiation-induced changes in the operating characteristics of bipolar transistor due to $1.2 \mathrm{MeV} \gamma$-ray irradiation in the dose range $0-7 \times 10^{5} \mathrm{rad}(1 \mathrm{rad}=0.01 \mathrm{~J} / \mathrm{kg})$ were performed. The collector, base and emitter currents as well as the current gain were calculated.

The output characteristics of bipolar transistor including the simulated and experimental results at the base current $10 \mu \mathrm{A}$ both for the initial transistor and for those irradiated by $1.2 \mathrm{MeV} \gamma$-rays with the doses $\Phi$ of $5 \times 10^{4}$ and $10^{5}$ rad are shown in Fig. 1. As can be seen, the collector current of irradiated transistor is several times smaller than the initial one. In the range of the collector voltage from $-5 \mathrm{~V}$ to $-15 \mathrm{~V}$, the simulated collector current correlates well with the experimental one, but the lower and higher collector voltages cause a small divergence of the simulated and experimental output characteristics.

Figure 2 shows the results of simulation and the experimental data of changes in the current gain of transistors during the irradiation depending on the $\gamma$-radiation dose in the range from $0-7 \times 10^{5} \mathrm{rad}$. The simulation shows strong dependence of the current gain on the base current and the radiation dose. Depending on the base current the value of the current gain can drop several times as compared with the initial one. For example, in the case of the base current $10 \mu \mathrm{A}$, the current gain is four times lower than the initial one with the $\gamma$-radiation dose 


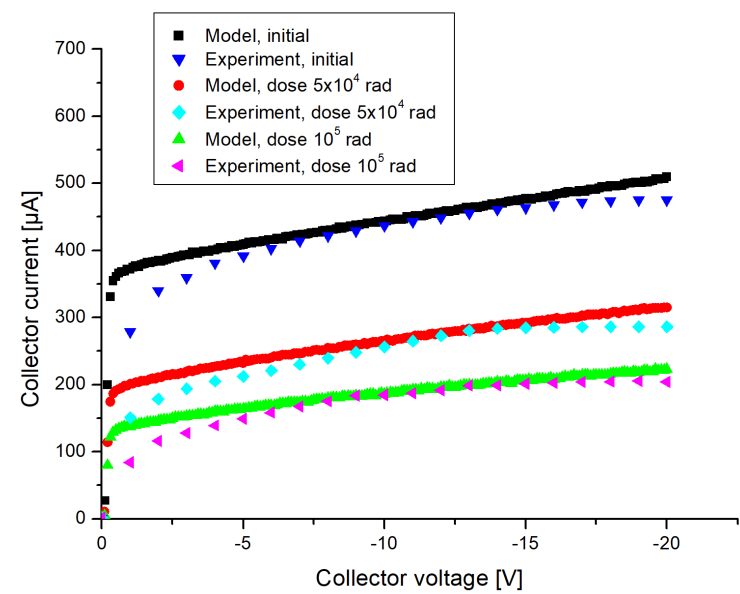

Fig. 1. Simulated and experimental output characteristics of initial and radiated bipolar transistor due to $1.2 \mathrm{MeV} \gamma$-rays with the doses of $5 \times 10^{4}$ and $10^{5} \mathrm{rad}$.

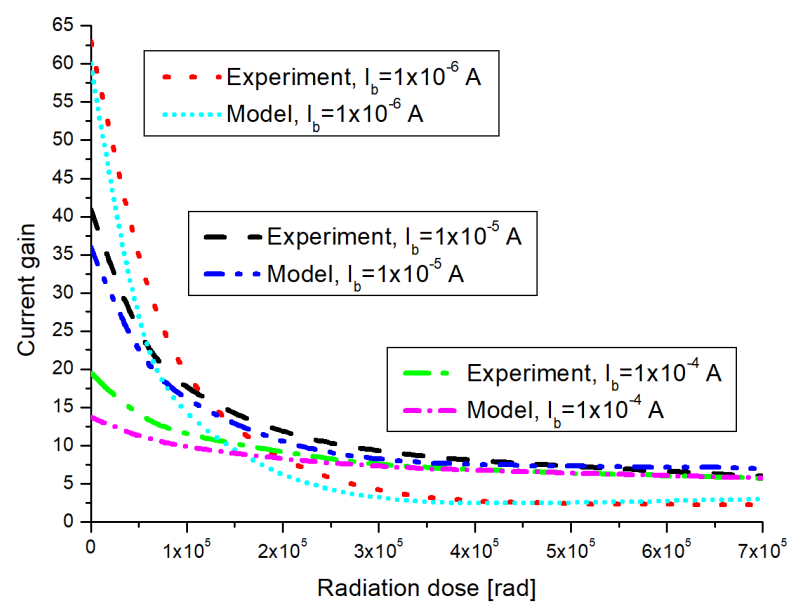

Fig. 2. Dose dependence of current gain of bipolar transistor due to $1.2 \mathrm{MeV} \gamma$-rays.

$3 \times 10^{5} \mathrm{rad}$. The same radiation dose provokes the decrease of current gain below twice as much as in the case of the base current $100 \mu \mathrm{A}$.

The dependence of current gain on the radiation dose, shown in Fig. 2, is monotonic and strong enough in the dose range lower than $3 \times 10^{5} \mathrm{rad}$ only. The further increase of the radiation dose leads to the weaker dependence. This can be explained taking into account the hyperbolic nature of the dependence $\tau(\Phi)$.
The transistor operating in the high-base-current mode is more radiation-resistant than that operating in the low-base-current mode, but its efficiency as a signal amplifier is lower as it is shown in Fig. 2.

The drop in the collector current during the irradiation indicates a significant increase in the recombination losses of the nonequilibrium charge carriers representing the base current, related to the shortening lifetime of the nonequilibrium charge carriers. The lifetime reduction is the result of radiation-induced defect formation in the operating device regions [8].

\section{Conclusions}

The physical and mathematical models of radiation degradation of the integral silicon bipolar transistors under the $1.2 \mathrm{MeV} \gamma$-ray irradiation in the dose range $0-7 \times 10^{5}$ rad were developed. The models can be used to calculate the radiation-induced changes in the output characteristics and the current gain. The results of simulation show the significant drop of output current that can be the cause of device malfunction. The obtained simulation results correlate well with the experimental data in the required range of the output voltage.

\section{References}

[1] T.R. Oldham, IEEE NSREC Short Course Notebook, 2011.

[2] A.N. Belous, V.A. Solodukha, S.V. Shveda, Space Electronics, Technosphera, Moscow 2015.

[3] J.R. Srour, C.I. Marshall, P.W. Marshall IEEE Trans. Nucl. Sci. 30, 653 (2003).

[4] A. Baschirotto, R. Castello, C. Onado, G. Pessina, P.G. Rancoita, A. Seidman Nucl. Instrum. Methods Phys. Res. B 122, 73 (1997).

[5] S. Miskiewicz, F. Komarov, A. Komarov, P. Zhukowski, V. Michailov Przeglad Elektrotechniczny 92, 11 (2016) (in Polish).

[6] E. Vologdin, A. Lysenko, Radiation Resistance of the Bipolar Transistors, Moscow 2000.

[7] E. Vologdin, A. Lysenko, Integral Radiation Changes of Semiconductor Parameters, Moscow 1999.

[8] K. Seeger, Physics of Semiconductors, Mir, Moscow 1977. 